\title{
Biologics: the role of delivery systems in improved therapy
}

\author{
This article was published in the following Dove Press journal: \\ Biologics:Targets and Therapy \\ 19 March 2014 \\ Number of times this article has been viewed
}

\author{
Nataša Škalko-Basnet \\ Drug Transport and Delivery Research \\ Group, Department of Pharmacy, \\ University of Tromsø, Tromsø, Norway
}

\begin{abstract}
The beginning of the 21 st century saw numerous protein and peptide therapeuticals both on the market and entering the final stages of clinical studies. They represent a new category of biologically originated drugs termed biologics or biologicals. Their main advantages over conventional drugs can be summarized by their high selectivity and potent therapeutic efficacy coupled with limited side effects. In addition, they exhibit more predictable behavior under in vivo conditions. However, up to now most of the formulations of biologics are designed and destined for the parenteral route of administration. As a consequence, many suffer from short plasma half-lives, resulting in their frequent administration and ultimately poor patient compliance. This review represents an attempt to address some of the challenges and promises in the product development of biologics both for parenteral and noninvasive administration. Some of the products currently in the pipeline of pharmaceutical development and corresponding perspectives are discussed in more detail.
\end{abstract}

Keywords: biologics, drug delivery systems, medical devices

\section{Introduction}

Biologics represent a new category of drugs which has rapidly gained momentum in the past decade. ${ }^{1}$ Although the first products were mostly insulin-related, more and more proteins and peptides are appearing in both research laboratories and pharmaceutical and biotechnological industries. Antibodies and their derivatives, particularly human monoclonal antibodies, are a rapidly growing category of targeted therapeutic agents. ${ }^{2}$ In addition, small interfering RNA, cytokines, enzymes, and a variety of peptide drugs are among the most studied biologicals. Rapid discoveries of new drug targets, more effective engineering processes, and knowledge on the fate of biologics in the body resulted in an increased number of biologics being on the market or in the late phases of clinical testing. ${ }^{3,4}$ Although manufactured by rather advanced technologies, most of these proteins and peptides are delivered via the "old fashioned" parenteral route, bearing all limitations linked to invasive drug delivery. ${ }^{5}$ As most of the therapy targets for biologics are chronic diseases, the limitations of invasive delivery are even more pronounced.

Protein therapeutics offer a highly specific and rather complex set of functions, limited interference with the normal biological processes, low immunogenicity, potential to replace gene therapy, and, from an industrial point of view, faster clinical development and approval time as well as better patent protection. ${ }^{2,6}$

Currently, there are about 200 therapeutic proteins on the market, of which about $10 \%$ have been rationally designed in respect to their pharmacokinetics. ${ }^{3}$ For example,
Correspondence: Nataša Škalko-Basnet Drug Transport and Delivery Research Group, Department of Pharmacy, University of Tromsø, Universitetsveien 57, 9037 Tromsø, Norway

Tel +4777 646640

Fax +47 77646 I5 I

Email natasa.skalko-basnet@uit.no 
over 20 monoclonal antibodies have been approved by the US Food and Drug Administration and the European Medicines Agency and are currently the fastest growing category of targeted therapeutic agents and are expected to retain the same attractiveness. ${ }^{2,7}$ In parallel, the fact that an increasing number of protein-based biologics are coming off patents in the next few years led to an extensive search for new products or formulations that can be patented and has been driving pharmaceutical industries to extensively focus on product development. ${ }^{8,9}$

New technologies enabling the improved delivery of biologics, such as needle-free devices, nanoparticles, and smart nanomaterials, together with the introduction of the concept of personalized medicine resulted in their faster market growth. ${ }^{7,10}$ One of the most challenging lines is the development of targeted delivery systems for small interfering RNA, which has up to now exhibited superiority under in vitro conditions but failed to achieve targeted as well as intracellular efficient delivery in various in vivo studies. ${ }^{4}$ Advanced delivery strategies could provide improvements in the targeted delivery of RNA drugs, enabling maximized drug potency while minimizing off-target toxicity and immunogenicity. ${ }^{11}$

As for all drug therapies, the efficient and targeted delivery of biologics to the desired site of action is the ultimate goal. However, due to their unique features, biologics represent a specific challenge in formulation development. Most often, the main strategies used in the product development of small molecular weight drugs cannot be readily transferred into the product development of biologics. The modified/ improved strategies need to be applied to face the specific challenges linked to protein and peptide drugs. In addition, specific challenges and opportunities in nonclinical safety testing of biologics need to be addressed and optimized. ${ }^{12}$

\section{Challenges of product development for biologics Challenge related to route of administration}

Most of the products currently on the market are designed for the parenteral route of administration. For example, monoclonal antibody drug products have been on the market for over 20 years and are still administered in an acute care setting through intravenous infusion. ${ }^{13}$ Some of these products are designed for self-injection by patients, mostly as subcutaneous injections. Many biologics would greatly benefit from administration via alternative routes. For example, limitations exhibited by human growth hormone may be overcome by alternative routes to currently applied subcutaneous injections which would not only increase the compliance and convenience to the patients but also assure the required dosing accuracy, very often an issue with subcutaneous administration. ${ }^{14}$

\section{Properties of formulations destined for parenteral administration}

In addition to being invasive, the parenteral route of administration also bears several limitations in regard to product development. One of the main concerns in formulation development is the exponential relationship between the concentrations of biologics and viscosity of the formulation; the highly viscous formulations, often required to assure desired concentration, cannot be readily injected. In addition, the $\mathrm{pH}$ and osmolality of the formulation need to be considered. The rapid development of nanotechnology, nanomedicine, and numerous delivery systems such as micro- and nanoparticles opened the possibilities to benefit from specially designed lipid- or polymer-based protein carriers. ${ }^{15}$ However, some of the polymeric particles used as protein carriers are designed to release the incorporated molecules by the diffusion-controlled mechanism, which, as a consequence, often requires particles in the micron range rather than nanoparticles and subsequently the use of larger gauge needles (21 gauge) for their administration. ${ }^{16}$

\section{Optimization of the dosage regimen}

Pharmacokinetic limitations mentioned earlier result in a need for both frequent drug injections and injection of rather large doses. ${ }^{13}$ For small molecular weight drugs the oral route is the preferable route of administration; however, biotherapeutics barely pass the gastrointestinal (GI) tract due to their instability. Improvements were achieved using mucoadhesive intestinal patches ${ }^{17}$ and nanotechnology-based devices and with the development of cell-penetration peptides. Cellpenetration peptides represent a mediated carrier for the oral delivery of biologicals; however, their safety during longterm application still needs to be confirmed. ${ }^{18}$

Sustained release formulations are expected to provide greater safety and therapeutic efficacy, consequently assuring reduced frequency of administration and ultimately improved patient compliance. ${ }^{16}$ In the case of biologics, this can be achieved through chemical modification and redesign of the molecule and de facto formation of a new chemical entity, which would require de novo registration as well. The pharmaceutical industry will therefore opt for encapsulation technologies as the 
preferable approach in product development. However, these technologies are often linked to increased manufacturing costs and the cost-effectiveness of the whole product development needs to be considered.

The development of oral formulations remains to be an unmet challenge. ${ }^{3}$

To improve the delivery of biologics via the commonly used parenteral route, two main strategies have been employed, namely 1) post-translational modification/conjugation and genetic fusion and 2) development of depot sustained release formulations of unmodified proteins with specific pharmacokinetic features. ${ }^{9}$

In this review, the focus is put on the development of sustained release formulations and the readers are advised to refer to Richard ${ }^{9}$ for an extensive overview of protein engineering and its potential in improved delivery of biologics.

Optimization of the dosage regimen for biologics requires multidisciplinary expertise due to the challenges in absorption, distribution, metabolism, and elimination (ADME). An overview of ADME-related challenges was provided by $\mathrm{Xu}$ and Vugmeyster. ${ }^{7}$ Additional limitations of biologics' ADME in humans are linked to the fact that the target-mediated clearance and anti-drug antibody-mediated clearance for therapeutic biologics have been confirmed to be species dependent, and thus represents a limitation in transferring the data from animal studies to humans. ${ }^{7}$ The fate of biologics in the liver and liver clearance are not yet fully understood and potential toxicities still need to be addressed.

\section{Optimization of formulation}

Recently, a concept of three pillars has been proposed as a base for drug development within pharmaceutical industries. Addressing three main questions could lead to the design and optimization of advanced delivery systems and devices, enabling improved therapeutic outcome for biologics as well:

1. Does the compound reach the target organ(s) at the necessary concentration?

2. Does the compound bind to the target(s) in vivo with the coverage required for biological activity?

3. Does the compound exert the functional modulation of the target? ${ }^{7}$

Several lines of research have been proposed in this respect, such as needle-free injection devices and polymeric microstructured arrays for the potential delivery of proteins, nanomedicine, and nanotechnology. ${ }^{19}$ Some of these research lines will be discussed in more detail in the following sections.

\section{Safety}

Although impressive efforts were put into improving the inherent instability of many biologics, concerns related to their immunogenicity and off-target toxicity remain. Delivery systems are expected to improve the targeted delivery of biologics, resulting in the subsequent reduction of the therapeutic dose while maintaining efficacy. However, the immunogenicity of many biologics is still an issue. For human antibodies the immunogenicity rate of zero seems to be unachievable, yet the new targeted therapeutics based on monoclonal antibodies promise limited low immunogenicity. ${ }^{2}$ Similarly, promising results from clinical trials with RNA-based therapeutics indicate that synthetic delivery carriers as well as chemical modifications of RNA therapeutics may provide the required outcome. ${ }^{11}$ The targeted delivery of biologics may limit the safety concerns.

\section{Routes of administration}

The majority of biologics in use nowadays are administered via parenteral routes. In addition to all well-known limitations related to the parenteral route, more attention should be given to the problem of uncontrollable and undesirable biodistribution and the uncontrollable metabolism and elimination of biologics, often overlooked in reported studies. ${ }^{3}$

\section{Oral route}

The oral route of drug administration remains to be the ultimate goal of any new drug therapy, especially therapy targeting chronic treatment. Moreover, specific therapies, such as insulin therapy, would greatly benefit from mimicking the physiological fate of endogenous insulin; subcutaneous administration often fails to mimic the glucose homeostasis in normal subjects as the insulin is being delivered directly to the peripheral circulation rather than bypassing the liver., ${ }^{9,20}$ An additional challenge in the development of oral insulin formulations is the metabolic changes inflicted by disease or age of the patient, which exhibit consequent changes in the ADME of the product. ${ }^{21}$

Due to their chemical structure, biologics are susceptible to harsh conditions in the GI tract, particularly to strong acid and proteolytic enzymes. Biologics also exhibit poor permeability and consequently poor bioavailability. ${ }^{5}$ Moreover, the electrostatic repulsion between the negatively charged protein and mucus layer that limits the close contact of the protein drugs on the epithelium is another factor responsible for their poor absorption via the oral route due to their rapid clearance from the site. ${ }^{22}$ 
Different strategies have been employed to combat the biochemical and enzymatic barriers of the GI tract. One of the first approaches, based on the positive experiences with PEGylation of nanodelivery systems for synthetic drugs, was the polymer polyethylene glycol (PEG) modification of a protein molecule. $\mathrm{PEG}$, through steric hindrance, provides protection against the harsh conditions found in the GI tract. ${ }^{5}$ However, attention has to be given to the site within the protein molecule where the PEG molecule is being attached as it may hinder the targetability of the protein.

In recent years, particulate carrier systems have been proposed as able to protect encapsulated biologics against both the acidity and proteolytic enzymes in the GI tract. Although particulates provide efficient protection against acidity in the GI tract, protection from the three enzymes (trypsin, $\alpha$-chymotrypsin, and pepsin) remains crucial for effective oral delivery. Up to now, no single polymer used for the particulate carrier system was capable of protecting the encapsulated biologics from all three enzymes. Such capability to avoid the effects of proteolytic enzymes is dependent on the polymer architecture and might be solved by the current development in the field of polymer chemistry, as proposed by Thompson et $\mathrm{a}^{23}$ who developed a novel quaternized amphiphilic polyallylamine able to spontaneously form nanocomplexes with insulin at $\mathrm{pH}$ 7.4. In vitro studies showed that high complexation efficiency (up to 93\%) assured the protection of insulin against trypsin and pepsin, and reduced cytotoxicity. ${ }^{23}$

In regard to the enhancement of absorption through the intestinal epithelium, various approaches were proposed as a means for improved permeability of biologics. These include the absorption enhancers such as surfactants, use of polyamines, use of chitosan to open the intracellular tight junction and aid paracellular transport of proteins, and cellpenetrating peptides. Various liposomal formulations have also been studied as absorption enhancers for biologics:

1. Bilosomes were proposed as an enhancer for the oral delivery of cholera toxin B subunit for targeted oral immunization against hepatitis B. The toxin B subunits were conjugated to bilosomes and were shown to induce a similar antibody titer response as an intramuscular injection of an alum-based antigen when evaluated in vivo in a mice model. ${ }^{24}$

2. Liposomes containing bile salts and used as a delivery system for insulin exhibited improved anti-enzyme protection and enhanced permeation under in vitro conditions. ${ }^{25}$ However, their stability and efficacy in vivo remains unconfirmed.
3. Orally administered lectin-modified insulin-containing liposomes exhibited superiority over conventional liposomes in a diabetic mice model with, however, still physiologically unaccepted bioavailabilty. ${ }^{26}$ Similarly, pectin-liposome nanocomplexes, able to facilitate the intimate contact with mucosal surface, have shown improved absorption of calcitonin in a rat model. ${ }^{27}$

Although the systems described above have shown some potential with in vitro and animal testing, their limitations, such as low bioavailability, remain a challenge. ${ }^{27}$

To overcome the limitation of a short residence time on the intestinal site, the use of mucoadhesive polymers has been proposed. ${ }^{28}$ Polymers, such as chitosans, pectin, carbomers, thiomers, and their derivatives can be utilized to manufacture mucoadhesive delivery systems. ${ }^{29,30}$ Their advantage in regard to formulation development is that the conditions applied in their manufacturing are milder and more protein friendly than those applied in the manufacturing of poly(lactic-co-glycolic) acid (PLGA) or poly(lactic) acid (PLA) nanoparticles. ${ }^{31}$ Hydrogel systems were also proposed as a means to improve the delivery of peptide and proteins; however, most attempts failed to improve delivery at a significant level. ${ }^{5}$

Nanoparticles and advancement in nanotechnology hold a promise for safe and efficient delivery of insulin and other biologicals. It is worth mentioning that the field still lacks well-defined safety studies, particularly in the case of chronic therapy, such as in diabetes. ${ }^{32}$

\section{Intranasal route}

The nasal route offers several advantages over the parenteral route with respect to the delivery of protein and peptide based biopharmaceuticals. The advantages can be summarized as follows: 1) noninvasiveness; 2) highly vascularized and permeable mucosal surface; 3 ) avoidance of first-pass metabolism; 4) rapid pharmacokinetics; and 5) ease of administration. ${ }^{33}$

Although the intranasal route remains very promising for small molecular weight drugs, in regard to the delivery of proteins, ie, rather large molecules, several limitations need to be overcome in order to fully utilize this route. The mucosal and enzymatic barriers, rapid clearance, and low residence time are just some of the obstacles that still need to be overcome. ${ }^{34}$

The original approach has been proposed based on the use of absorption promoters such as surfactants, enzymatic inhibitors, or multifunctional polymers. ${ }^{14}$ This approach was followed by the extensive use of mucoadhesive 
delivery systems such as bioadhesive microspheres and chitosan-based delivery systems. However, the efficacy of these systems is significantly lower than parenterally administered systems and their main advantages have not been proven yet. ${ }^{14}$

A lipid-based colloidal delivery system, Pheroid ${ }^{\mathrm{TM}}$ technology (North-West University, Potchefstroom, South Africa), is a new strategy in the pipeline for nasal delivery of biologics. Due to the fact that this technology is based on the use of essential fatty acids, it has the potential to avoid safety issues related to polymer use. ${ }^{5}$ Pheroid technology consists of a unique submicron emulsion based on essential fatty acids. Pheroid vesicles and microsponges have been studied for nasal delivery of human growth factor and have showed promising absorption enhancement in a rat model, although not yet superior to chitosan-based absorption enhancers. ${ }^{35}$ Similar observations were made when the same technology was employed to deliver calcitonin both nasally and orally. Although the improvements were noticeable, chitosan-based enhancers were found to be superior, particularly with the nasal route. ${ }^{36}$

Moreover, nasal delivery is considered as an attractive route in delivery aimed at brain targeting and overcoming the blood-brain barrier. ${ }^{37}$ Intranasal delivery of large molecular weight biologics, such as proteins and gene vectors, is an attractive means to treat a variety of central nervous system disorders such as multiple sclerosis, Parkinson's disease, epilepsy, and Alzheimer's disease. ${ }^{38}$

\section{Pulmonary route}

Respiratory tract-targeted delivery also offers a noninvasive alternative both for local and systemic conditions. It provides rather fast onset and favorable pharmacokinetics, avoiding the harsh conditions of the GI tract and first-pass metabolism. ${ }^{39}$ The success of aerosol-based delivery of proteins and peptides will be dependent on physiological factors (eg, breathing pattern, alveolar macrophages) and specific properties of biologics (eg, molecular weight, lipophilicity). The protective mucus layer covering the airway epithelium is an additional barrier to drug absorption. The optimal particle properties of aerosols, such as size, size distribution, surface characteristics, and drug load, are the prerequisites for successful protein-based therapy. ${ }^{15}$ Although the failure of Exubera ${ }^{\circledR}$ (short-acting insulin-loaded dry powder inhalers system; Pfizer, Inc., New York, NY, USA) slowed the development of delivery systems via the pulmonary route, several promising lines might overcome the current limitations of this route, as summarized in a review by Chung et al. ${ }^{5}$

\section{Transdermal route}

The transdermal route of drug delivery is based on the passive diffusion of molecules through the stratum corneum and is, therefore, directly dependent on the drug's size, physicochemical properties, and susceptibility to metabolism by skin enzymes. The emerging strategies for the transdermal delivery of peptide and protein drugs are summarized in an extensive review by Schuetz et al. ${ }^{40}$ Several delivery systems have been proposed to serve as optimal carriers for the percutaneous delivery of growth factors; ${ }^{41}$ however, their efficacy under in vivo conditions remains to be confirmed.

A lyophilized hydrogel patch system was developed for microneedle-mediated insulin delivery. The system demonstrated an ability to provide an appropriate reservoir for the sustained release of insulin via microneedle-mediated transdermal delivery in animal studies with diabetic rats. ${ }^{42}$

A vaginal route of drug administration is gaining attention as an alternative route of drug administration, including the delivery of proteins and peptides. ${ }^{43}$

\section{Delivery of therapeutic proteins via the parenteral route}

The main advantage of focusing on the development of sustained release biologics formulations to be administered by the parenteral route rather than on the use of protein engineering is the avoidance of any chemical or fusion modification of the protein. However, one should keep in mind that the success seen in the development of injectable depot formulations for small molecules and peptides cannot be readily reformulated into products for protein-based biologicals. $^{9}$

The main systems used in the development of sustained release formulations for proteins can be summarized as: 1) liquid gelling or self-assembling systems; 2) biodegradable micro- and nanoparticles based on polymers such as PLA or PLGA; 3) lipid-based systems; 4) protein microcrystals; 5) micro- and nanoparticles made of hydrophilic or amphiphilic polymers; and 6) biodegradable or nonbiodegradable but removable solid implants.

\section{Liquid gelling or self-assembling systems}

A system composed of a water soluble triblock copolymer (PLGA-PEG-PLGA), exhibiting low viscosity at room temperature and high viscosity when injected, provided sustained release of the protein. In rat experiments with porcine growth hormone, it assured sustained release of protein over a 15-day period. ${ }^{9}$ Another promising system is based on 
a highly viscous component, sucrose acetate isobutyrate, which enables injection through a 23- or 25-gauge needle. Several other systems based on Pluronic ${ }^{\circledR}$ (BASF Corporation New Jersey, NJ, USA) triblock polymers complexed with short PLA chains or photopolymerizable hydrogels as well as alginate- or dextran-based polysaccharides seem to be showing the expected improvements in the preclinical studies. ${ }^{44,45}$

\section{Biodegradable micro- and nanoparticles based on polymers such as PLA or PLGA}

Multiblock Pluronic copolymers linked by D-lactide and L-lactide oligomers of different spacer lengths enabled formation of the physically crosslinked Pluronic hydrogels, exhibiting significantly altered sol-gel phase transition behavior with superior critical gelation concentrations and temperatures. Moreover, newly synthesized hydrogels provided increased mechanical strength with high resistance to rapid dissolution in aqueous medium. When the system was used to deliver human growth hormone, it prolonged the release of the hormone for up to 13 days under in vitro conditions. The release was based on the diffusion/erosion coupled mechanism and followed zeroorder pharmacokinetics. Physically crosslinked hydrogels provide a friendly environment in regard to the protection of proteins and peptides as compared to chemically crosslinked hydrogels. ${ }^{44}$

\section{Biodegradable polymer microparticles}

Although PLGA microspheres were a popular carrier for various types of small molecules, the first product based on PLGA microspheres - developed for human growth hormone and approved in 1999 - had to be removed from the market in 2004 for reasons not yet fully justified, though mostly attributed to high costs of manufacturing. ${ }^{9,14}$ A new technology, based on liquefying PLGA in the presence of supercritical carbon dioxide and avoiding the use of any organic solvent, has been developed. ${ }^{46}$ However, the use of PLGA copolymers remains limited due to poor compatibility of the proteins with PLGA copolymers. ${ }^{9}$ To overcome this compatibility issue, a new biodegradable polymer has been introduced for the production of protein-containing microspheres. ${ }^{47}$ Poly(ether-ester) multiblock copolymers composed of PEG terephthalate and poly(butylene terephthalate) exhibited superior controlled release properties; however, its biocompatibility was found to be dependent on the size and shape of the implant, as evaluated in rabbit studies. ${ }^{47}$ The additional advantage of the copolymer is that its microspheres are suitable for sterilization via gamma irradiation. ${ }^{47}$

\section{Solid lipid microparticles and microcapsules}

Lipid microparticles loaded with insulin were shown to release insulin over $>14$ days in an in vitro study. ${ }^{15}$ Similarly, the spray congealing used to produce glycerol tripalmitate microparticles loaded with a high amount of insulin enabled its release over 28 days under in vitro conditions. ${ }^{48}$ Solid lipid microcapsules containing a solid human growth hormone in its inner core achieved sustained release of human growth hormone over 7 days and exhibited strong improvement of the apparent protein half-life. The same type of microcapsules could be prepared by the pressurized fluid process at lower temperatures $\left(60^{\circ} \mathrm{C}\right)$. These lipid-based systems were prepared by the avoidance of solvent during the manufacturing and may, therefore, be promising for protein delivery. ${ }^{9}$ However, their clinical evaluation needs to be performed.

\section{Water-based particulate systems}

This approach mostly utilizes polyelectrolyte-coated protein microcrystals dispersed in aqueous vehicle for injection, or hydrogels. ${ }^{9}$ The nanoparticulate Medusa ${ }^{\circledR}$ formulation developed by Flamel Technologies (Vénissieux, France) is based on the poly-L-glutamate backbone grafted with hydrophobic $\alpha$-tocopherol molecules creating a colloidal dispersion of nanoparticles $(10-50 \mathrm{~nm})$ in water. ${ }^{49}$ The particle dispersion forms a subcutaneous gel-like implant after subcutaneous injection, providing a depot for protein release. Some of these formulations are already in Phase II clinical trials. ${ }^{9}$ Up to now, the most successful formulation of this type consists of sodium hyaluronate microparticles produced by spray-drying technology. The results of a 3-year Phase II clinical trial conducted globally and involving 167 pediatric patients confirmed the efficacy and safety of the system containing LB03002. ${ }^{50}$

\section{Crystalline biopharmaceuticals}

Although this approach in formulation development offers an advantage of avoiding alterations in the original protein structure, in general the high intrinsic solubility of proteins may seem to be counterintuitive. Crystals of recombinant human growth hormone coated with a monomolecular layer of positively charged polyarginine provided in vivo pharmacokinetic release profiles of several days in animal models. Moreover, the noninvasive nature of the suspension facilitated easy administration through a 30 -gauge needle. ${ }^{16}$

\section{Solid implants}

Solid implants were also designed and developed to achieve release of proteins over a longer period of time, 
usually 3-12 months. ${ }^{9}$ Duros ${ }^{\circledR}$ delivery technology (Durect Corporation, Cupertino, CA, USA), consisting of sterile, nonbiodegradable single-use solid implants is an example of such a system. The system provides controlled protein release over 3-12 months and can be removed at any time during the therapy quickly and safely. Several of the implants developed by this technology have been proven successful in Phase I, II, and III clinical studies. ${ }^{9}$

\section{New delivery devices}

In response to the high incidence of discontinuation of treatment due to noncompliance, several new delivery devices have been proposed such as prefilled syringes, manual injector pens, autoinjectors, and needle-free devices. ${ }^{13,51}$ Significant improvements were also made in the field of micro/nanomechanical device-based drug delivery. These devices have shown potential in developing carriers with controlled physicochemical properties (especially size). ${ }^{19}$

These delivery devices particularly target the pediatric population of patients, and seem to be providing the confirmed advantages of needle-based technologies. It is expected that more technological improvements will be obtained in this still not fully explored area. In particular, devices for special populations of patients, such as children, elderly, and arthritic patients, need to become the focus of further development.

\section{Conclusion}

Biological origin drugs are becoming increasingly present in pharmaceutical development. Although the manufacturing of biologics has exhibited certain progress, particularly in the past few years, progress in the development of delivery systems able to improve the bioavailability of biologics remains rather limited. Most of the products still require administration via an invasive route. New technologies and new approaches in designing delivery systems for biologics might lead to faster product development in this exciting part of pharmaceutical development.

\section{Disclosure}

The author reports no conflict of interest in this work.

\section{References}

1. Kling J. Fresh from the biotech pipeline - 2011. Nat Biotechnol. 2012;30(2):128-131.

2. Nelson AL, Dhimolea E, Reichert JM. Development trends for human monoclonal antibody therapeutics. Nat Rev Drug Discov. 2010;9(10): 767-774.

3. Ezan E. Pharmacokinetic studies of protein drugs: past, present and future. Adv Drug Deliv Rev. 2013;65(8):1065-1073.
4. Poelstra K, Beljaars L, Melgert BN. Cell-specific delivery of biologicals: problems, pitfalls and possibilities of antifibrotic compounds in the liver. Drug Discov Today. 2013;18(23-24):1237-1242.

5. Chung SW, Hil-lal TA, Byun Y. Strategies for non-invasive delivery of biologics. J Drug Target. 2012;20(6):481-501.

6. Leader B, Baca QJ, Golan DE. Protein therapeutics: a summary and pharmacological classification. Nat Rev Drug Discov. 2008;7(1):21-39.

7. Xu X, Vugmeyster Y. Challenges and opportunities in absorption, distribution, metabolism and excretion studies of therapeutic biologicals. AAPS J. 2012;14(4):781-791.

8. Martin-Moe S, Lim FJ, Wong RL, Sreedhara A, Sundaram J, Sane SU. A new roadmap for biopharmaceutical drug product development: integrating development, validation and quality by design. $J$ Pharm Sci. 2011;100(8):3031-3043.

9. Richard J. Parenteral biologics delivery: recent progresses, key challenges and perspectives. European Journal of Parenteral and Pharmaceutical Sciences. 2012;17(3):94-109.

10. Duncan R, Gaspar R. Nanomedicine(s) under the microscope. Mol Pharm. 2011;8(6):2101-2141.

11. Burnett JC, Rossi JJ. RNA-based therapeutics: current progress and future prospects. Chem Biol. 2012;19(1):60-71.

12. Kronenberg S, Baumann A, de Haan L, et al. Current challenges and opportunities in nonclinical safety testing of biologics. Drug Discov Today. 2013;18(23-24):1138-1143.

13. Burgess BE. Optimizing drug delivery for modern biologics. BioPharm International. 2012;25(5):30-32.

14. Cázares-Delgadillo J, Ganem-Rondero A, Kalia YN. Human growth hormone: new delivery systems, alternative routes of administration, and their pharmacological relevance. Eur J Pharm Biopharm. 2011;78(2): 278-288.

15. Reithmeier H, Hermann J, Gőpferich A. Lipid microparticles as a parenteral controlled release device for peptides. J Control Release. 2001;73(2-3):339-350.

16. Govardhan C, Khalaf N, Jung CW, et al. Novel long-acting formulation of human growth hormone. Pharm Res. 2005;22(9):1461-1470.

17. Whitehead K, Shen Z, Mitragotri S. Oral delivery of macromolecules using intestinal patches: applications for insulin delivery. $J$ Control Release. 2004;98(1):37-45.

18. Khafagy el-S, Morishita M. Oral biodrug delivery using cell-penetrating peptide. Adv Drug Del Rev. 2012;64(6):531-539.

19. Shi J, Votruba AR, Farokhzad OC, Langer R. Nanotechnology in drug delivery and tissue engineering: from discovery to applications. Nano Lett. 2010;10(9):3223-3230.

20. Sonaje K, Lin YH, Juang JH, Wey SP, Chen CT, Sung HW. In vivo evaluation of safety and efficacy of self-assembled nanoparticles for oral insulin delivery. Biomaterials. 2009;30(12):2329-2339.

21. Krol S, Ellis-Behke R. Marchetti P. Nanomedicine for treatment of diabetes in an aging population: state-of-the-art and future developments. Nanomedicine. 2012;8(Suppl 1):S69-S76.

22. Cone RA. Barrier properties of mucus. Adv Drug Deliv Rev. 2009;61(2): $75-85$.

23. Thompson CJ, Tetley L, Uchegbu IF, Cheng WP. The complexation between novel comb shaped amphiphilic polyallylamine and insulin: towards oral insulin delivery. Int J Pharm. 2009;376(1-2):46-55.

24. Shukla A, Katare OP, Singh B, Vyas SP. M-cell targeted delivery of recombinant hepatitis $\mathrm{B}$ surface antigen using cholera toxin $\mathrm{B}$ subunit conjugated bilosomes. Int J Pharm. 2010;385(1-2):47-52.

25. Niu M, Lu Y, Hovgaard L, Wu W. Liposomes containing glycocholate as potential oral insulin delivery systems: preparation, in vitro characterization, and improved protection against enzymatic degradation. Int J Nanomedicine. 2011;6:1155-1166.

26. Zhang N, Ping QN, Huang GH, Xu WF. Investigation of lectin-modified insulin liposomes as carriers for oral administration. Int $J$ Pharm. 2005;294(1-2):247-259.

27. Thirawong N, Thongborisute J, Takeuchi H, Sriamornsak P. Improved intestinal absorption of calcitonin by mucoadhesive delivery of novel pectinliposome nanocomplexes. J Control Release. 2008;125(3):236-245. 
28. Smart JD. The basics and underlying mechanisms of mucoadhesion. Adv Drug Deliv Rev. 2005;57(11):1556-1568.

29. Builders PF, Kunle OO, Okpaku LC, Builders MI, Attama AA, Adikwu MU. Preparation and evaluation of mucinated sodium alginate microparticles for oral delivery of insulin. Eur J Pharm Biopharm. 2008;70(6):777-783.

30. Dünnhaupt S, Barthelmes J, Hombach J, Sakloetsakun D, Arkhipova V, Bernkop-Schnürch A. Distribution of thiolated mucoadhesive nanoparticles on intestinal mucosa. Int J Pharm. 2011;408(1-2): 191-199.

31. George M, Abraham TE. Polyionic hydrocolloids for the intestinal delivery of protein drugs: alginate and chitosan - a review. $J$ Control Release. 2006;114(1):1-14.

32. Card JW, Magnuson BA. A review of the efficacy and safety of nanoparticle-based oral insulin delivery systems. Am J Physiol Gastrointest Liver Physiol. 2011;301(6):G956-G967.

33. Illum L. Nasal drug delivery - possibilities, problems and solutions. J Control Release. 2003;87(1-3):187-198.

34. Washington N, Washington C, Wilson CG, editors. Physiological Pharmaceutics, Barriers to Drug Absorption. 2nd ed. London: Taylor and Francis; 2001:199-220.

35. Steyn D, du Plessis L, Kotzé A. Nasal delivery of recombinant human growth hormone: in vivo evaluation with Pheroid technology and N-trimethyl chitosan chloride. J Pharm Pharm Sci. 2010;13(2): 263-273.

36. Du Plessis LH, Lubbe J, Strauss T, Kotzé AF. Enhancement of nasal and intestinal calcitonin delivery by the novel Pheroid fatty acid based delivery system, and by $\mathrm{N}$-trimethyl chitosan chloride. Int $J$ Pharm. 2010;385(1-2):181-186.

37. Dhuria SV, Hanson LR, Frey WH 2nd. Intranasal delivery to the central nervous system: mechanism and experimental considerations. JPharm Sci. 2010;93(4):1654-1673.

38. Lochhead JJ, Thorne RG. Intranasal delivery of biologics to the central nervous system. Adv Drug Del Rev. 2012;64(7):614-628.

39. Agu RU, Ugwoke MI, Armand M, Kinget R, Verbeke N. The lung as a route for systemic delivery of therapeutic proteins and peptides. Respir Res. 2001;2(4):198-209.

40. Schuetz YB, Naik A, Guy RH, Kalia YN. Emerging strategies for the transdermal delivery of peptide and protein drugs. Expert Opin Drug Deliv. 2005;2(3):533-548.
41. Jeon SO, Hwang HJ, Oh DH, et al. Enhanced percutaneous delivery of recombinant human epidermal growth factor employing nano-liposome system. J Microencapsul. 2012;29(3):234-241.

42. Qiu Y, Qin G, Zhang S, Wu Y, Xu B, Gao Y. Novel lyophilized hydrogel patches for convenient and effective administration of microneedlemediated insulin delivery. Int J Pharm. 2012;437(1-2):51-56.

43. Vanić Ž, Škalko-Basnet N. Nanopharmaceuticals for improved topical vaginal therapy: can they deliver? Eur J Pharm Sci. 2013;50(1): $29-41$.

44. Chung HJ, Lee Y, Park TG. Thermo-sensitive and biodegradable hydrogels based on stereocomplexed Pluronic multi-block copolymers for controlled protein delivery. J Control Release. 2008;127(1): 22-30.

45. Van Tomme SR, Storm G, Hennink WE. In situ gelling hydrogels for pharmaceutical and biomedical applications. Int $J$ Pharm. 2008;355(1-2):1-18.

46. Jordan F, Naylor A, Kelly CA, Howdle SM, Lewis A, Illum L. Sustained release hGH microsphere formulation produced by a novel supercritical fluid technology: in vivo studies. J Control Release. 2010;141(1): 153-160.

47. van Dijkhuizen-Radersma R, Hesseling SC, Kaim PE, de Groot K, Bezemer JM. Biocompatibility and degradation of poly(etherester) microspheres: in vitro and in vivo evaluation. Biomaterials. 2002;23(24):4719-4729.

48. Maschke A, Becker C, Eyrich D, Kiermaier J, Blunk T, Göpferich A. Development of a spray congealing process for the preparation of insulin-loaded lipid microparticles and characterization thereof. Eur $J$ Pharm Biopharm. 2007;65(2):175-187.

49. Chan YP, Meyruiex R, Kravtzoff R, Nicolas F, Lundstrom K. Review on Medusa ${ }^{\circledR}$ : a polymer-based sustained release technology for protein and peptide drugs. Expert Opin Drug Deliv. 2007;4(4):441-451.

50. Peter F, Bidlingmaier M, Savoy C, Ji HJ, Saenger PH. Three-year efficacy and safety of LB03002, a once-weekly sustained-release growth hormone $(\mathrm{GH})$ preparation, in prepubertal children with GH deficiency (GHD). J Clin Endocrinol Metab. 2012;97(2):400-407.

51. Dumas H, Panayiotopoulos P, Parker D, Pongpairochana V. Understanding and meeting the needs of those using growth hormone injection devices. BMC Endocr Disord. 2006;6:5.
Biologics: Targets \& Therapy

\section{Publish your work in this journal}

Biologics: Targets \& Therapy is an international, peer-reviewed journal focusing on the patho-physiological rationale for and clinical application of Biologic agents in the management of autoimmune diseases, cancers or other pathologies where a molecular target can be identified. This journal is indexed on PubMed Central, CAS, EMBase, Scopus

\section{Dovepress}

and the Elsevier Bibliographic databases. The manuscript management system is completely online and includes a very quick and fair peerreview system, which is all easy to use. Visit http://www.dovepress. com/testimonials.php to read real quotes from published authors. 